\title{
Laser active pyrometry and lock-in thermography for characterization of deposited layers on plasma facing components from European tokamaks
}

\author{
by A. Semerok*, S.V. Fomichev** and C. Grisolia***
}

\author{
* CEA, DEN, DPC/SEARS/LISL, F-91191 Gif-sur-Yvette, France, alexandre.semerok@cea.fr \\ ** NRC Kurchatov Institute, 123182 Moscow, Russia, svfomichev@mail.ru \\ *** CEA, IRFM, F-13108 Saint-Paul-lez-Durance, France, christian.grisolia@cea.fr
}

\begin{abstract}
Laser active pyrometry and lock-in thermography for characterization of micrometric layers on plasma facing components from European tokamaks have been under experimental and theoretical studies in CEA (France). The samples with a deposited layer of different thickness $\sim(1-100) \mu \mathrm{m}$ were under heating by a Nd-YAG repetition rate laser $(1 \mathrm{~Hz}-10 \mathrm{kHz})$. A pyrometer system was used for temperature measurements in $(500-2600) \mathrm{K}$ range. For both methods, the layer characterization was provided by the best fit of the simulation data with the experimental ones. The computer simulations were performed with the developed theoretical models. Some practical applications and possible improvements of the methods will be discussed.
\end{abstract}

\section{Introduction}

Laser active thermography methods for non-destructive testing and diagnostics have found their applications in different fields of industry, technology, and arts. For nuclear field, the methods may offer the additional advantage of automated remote control and tests of nuclear installations without personnel attendance in contaminated zone. In controlled thermo-nuclear fusion, a number of technological problems should find their solution to provide an appropriate functioning of installations. Formation of deposited layers with a high content (up to $50 \%$ ) of hydrogen isotopes (D and T) on plasma facing component (PFC) may be mentioned as one of the problems. A friable carbon deposited layer is a source of micrometric dust which increases fusion plasma losses. High tritium content is also quite undesirable for personnel radiation exposure and nuclear safety. The future use of new materials such as tungsten, beryllium and carbon fiber composite (CFC) with tungsten protective layer makes the development of in situ diagnostics by thermography methods of a vital importance.

In these methods, the surface under analysis suffers a repetition rate laser heating. Based on thermal response of the heated surface, one may get information on its mechanical properties (thickness, under surface defects and cracks) and also on thermo-physical features (thermal conductivity and diffusivity, thermal resistance coefficient of the layer/surface interface). Surface characterization is provided by comparison and best fit of the simulated results (heating temperatures or phase shifts) obtained by the adequate theoretical models with the experimental data.

In search for appropriate in situ non-destructive diagnostics, laser active pyrometry and lock-in thermography have been under experimental and theoretical studies in CEA (France) [1-8]. Here, theoretical studies will be presented with respect to the experimental investigations on PFCs with deposited layers from European tokamaks. For heating temperature calculation, two approaches (direct numerical finite-difference solution and analytical solution followed by calculations) may be used. In a general case with the temperature-dependent parameters and with a moving laser beam, it can be made only by direct space-time (3+1 dimensional) numerical simulations by the finite-difference methods. With the direct numerical solution, it is easy to take into account the temperature dependence of the thermo-physical and optical parameters. However, even for 1D case, it is difficult to trace the heating temperature for both long time intervals and a long spatial scale. In the case of constant thermo-physical parameters, the analytical expressions for laser heating of complex surfaces can be deduced and analyzed. With the analytical solution approach, it is easy to take into account $3 \mathrm{D}$ properties and to trace them for a long time and on a long spatial scale.

The solutions obtained for the periodical laser heating and the method developed for direct phase shift calculation were applied for simulations of the experimental data obtained by laser active pyrometry and lock-in thermography.

\section{Layer heating by repetition rate laser beam}

The models for laser heating of complex surfaces with a micrometric layer $(1-1000 \mu \mathrm{m})$ on a substrate were developed in [1-2, 6-8]. The calculations of the surface temperature under laser heating are generally related with a complex nonlinear thermo-physical problem associated with the temperature dependence of the thermo-physical and optical properties of the surface. The standard heat equations [9] for the surface $(s)$ with a layer $(I)$ of thickness $d$ and with an arbitrary heat transfer coefficient $(h>0)$ for the layer/substrate interface are as follows: 


$$
\begin{array}{ll}
c_{l}(T) \frac{\partial T}{\partial t}=\operatorname{div}\left(k_{l}(T) \nabla T\right)+Q_{l}(t, r, z), & 0<\mathrm{z}<d, \\
c_{S}(T) \frac{\partial T}{\partial t}=\operatorname{div}\left(k_{s}(T) \nabla T\right)+Q_{s}(t, r, z), & z>d .
\end{array}
$$

The boundary conditions on the layer/substrate interface should reflect the continuity of the flux of the thermal energy through the surface $z=d$. It can be written as:

$$
-\left.k_{l}(T) \frac{\partial T(t, \vec{r})}{\partial z}\right|_{z=d-0}=-\left.k_{s}(T) \frac{\partial T(t, \vec{r})}{\partial z}\right|_{z=d+0} \equiv q_{d} .
$$

To take into account the heat resistance between the layer and the substrate, the boundary condition relating the finite temperature jump and the heat flux on the interface should be introduced:

$$
\left.\Delta T_{d} \equiv T(t, \vec{r})\right|_{z=d-0} \quad-\left.T(t, \vec{r})\right|_{z=d+0}=\frac{q_{d}}{h} .
$$

The consideration of the heat resistance requires introducing the second interface layer between the first boundary layer and the substrate, where the heat flux could be inhibited, and the interface temperature jump corresponds to the temperature difference on the different sides of the second layer. However, such approach requires also introducing more adjusting parameters. Because of a poor knowledge about the physical-chemical properties of the layer/substrate interface, it is more reasonable to describe it by phenomenological relation (3) with the only adjusting parameter $h$.

The boundary condition at $z=0$ (at the interface between the layer and the ambient air) should be added to complete the statement of the problem. It can be written as:

$$
\left.\frac{\partial T(t, \vec{r})}{\partial z}\right|_{z=0}=\mu\left(\left.T(t, \vec{r})\right|_{z=0}-T_{0}\right),
$$

where $\mu$ is the convective heat transfer coefficient and $T_{0}$ is the ambient air temperature. In our experimental conditions we can safely assume that $\mu=0$.

The initial condition to the system of equations (1) will always be supposed to be of the form $T(t=0, \vec{r})=T_{0}$, where $t=0$ is the time moment of the laser switching on. be written as

Laser heating source terms $Q_{l}(t, \vec{r})$ and $Q_{s}(t, \vec{r})$ are deduced from macroscopic electrodynamics [10] and can

$$
\overline{Q_{l}(t, \vec{r})}=f_{Z}^{l}(z) f_{X Y}(x-v t, y) f_{T}(t), \quad Q_{s}(t, \vec{r})=f_{Z}^{s}(z) f_{X Y}(x-v t, y) f_{T}(t),
$$

where $f_{X Y}(x, y)$ and $f_{T}(t)$ are the spatial and temporal laser beam profiles, $v[\mathrm{~m} / \mathrm{s}]$ is the laser beam scanning velocity along the $x$-axis, and

$$
\begin{aligned}
& f_{Z}^{\prime}(z)=I_{0} \frac{\omega \varepsilon_{l}^{\prime \prime}}{4 c}\left|\tilde{C}_{s}\right|^{2}\left|1+\sqrt{\frac{\varepsilon_{s}}{\varepsilon_{l}}}\right|^{2}\left\{\exp \left(-\alpha_{l} z\right)+\left|\frac{1-\sqrt{\varepsilon_{s} / \varepsilon_{l}}}{1+\sqrt{\varepsilon_{s} / \varepsilon_{l}}}\right|^{2} \exp \left[\alpha_{l}(z-2 d)\right]\right\}, \\
& f_{Z}^{s}(z)=I_{0} \frac{\omega}{c} \varepsilon_{s}^{\prime \prime}\left|\tilde{C}_{s}\right|^{2} \exp \left[\alpha_{s}(d-z)-\alpha_{l} d\right], \\
& \tilde{C}_{s}=4\left\{\left(1+\sqrt{\varepsilon_{l}}\right)\left(1+\sqrt{\frac{\varepsilon_{s}}{\varepsilon_{l}}}\right) \exp \left(-\frac{i \omega d n_{l}}{c}\right)+\left(1-\sqrt{\varepsilon_{l}}\right)\left(1-\sqrt{\frac{\varepsilon_{s}}{\varepsilon_{l}}}\right) \exp \left(\frac{i \omega d n_{l}}{c}-\alpha_{l} d\right)\right\}^{-1},
\end{aligned}
$$

with $\varepsilon_{l}$ and $\varepsilon_{s}$ the complex dielectric permittivities of the layer and the substrate. With a good accuracy, we can safely average the source term $Q_{l}(t, r)$ inside the layer (that denotes in expression (4) by overlining) over the laser field spatial oscillations. Here, $I_{0}$ is the peak laser intensity, $c$ is the light velocity, $\omega$ is the frequency of the laser light, $\varepsilon_{l}^{\prime \prime}$ and $\varepsilon_{s}^{\prime \prime}$ are the imaginary parts of the complex dielectric permittivities connected with the corresponding absorption coefficients of the layer and the substrate by relations $\alpha_{l}=\omega \varepsilon_{l}^{\prime \prime} / c n_{l}$ and $\alpha_{s}=\omega \varepsilon_{s}^{\prime \prime} / c n_{s}$, with $n_{l}=\mathfrak{R}\left(\sqrt{\varepsilon_{l}}\right)$ and $n_{s}=\mathfrak{R}\left(\sqrt{\varepsilon_{s}}\right)$ the refraction coefficients of the laser light in the boundary layer and in the substrate, respectively. The light reflectivity coefficients on the interfaces between the corresponding medium (layer or substrate) and vacuum, namely,

$$
R_{l}=\frac{\left(n_{l}-1\right)^{2}+\kappa_{l}^{2}}{\left(n_{l}+1\right)^{2}+\kappa_{l}^{2}} \text { and } R_{s}=\frac{\left(n_{s}-1\right)^{2}+\kappa_{s}^{2}}{\left(n_{s}+1\right)^{2}+\kappa_{s}^{2}}
$$

are also expressed through the parameters connected with the dielectric permittivities $\varepsilon_{l}=\left(n_{l}+i \kappa\right)^{2}$ and $\varepsilon_{S}=\left(n_{S}+i \kappa_{S}\right)^{2}$.

\subsection{Numerical and analytical solutions}

The laser heating calculations in a general case (with the temperature-dependent parameters and with a moving laser) can be made only by direct space-time (3+1 dimensional) numerical simulations by the finite-difference methods. With the direct numerical solution, it is easy to take into account the temperature dependence of the thermo-physical and optical parameters. It should be regarded as an advantage of this method. However, it is difficult to trace the heating properties for both long time intervals and a long spatial scale even for 1D case, let alone 2D or 3D cases. It should be considered as a disadvantage of this method.

On the other hand, in the case of constant thermo-physical parameters the analytical expressions for laser heating of complex surfaces can be deduced and analyzed. With the analytical solution approach, it is easily to take into 
account 3D properties and to trace them for a long time and on a long spatial scale. This is an advantage of this method. The necessity to neglect the temperature dependence on the sample parameters should be considered as a disadvantage of this method.

Generally, for temperature-dependent optical and thermo-physical parameters of both the layer and the substrate, the solution of the equations (1) in 3D-space, together with the boundary conditions (2a-c) corresponds to a difficult non-linear problem even for moderate computers, especially if we are interested in the temperature distributions on large scales, both in space and time. For this reason, and also because of poor knowledge of the temperature dependences of some parameters for media under study, we will consider the analytical solution of the system of equations (1) assuming the mean constant values independent of the temperature (in the working range of temperatures between the ambient and the sublimation/evaporation temperatures) for all the optical and thermo-physical parameters involved in the problem. With the constant parameters, the problem under study is linear. Its solution can be obtained by the Fourier method. Omitting the details of the derivation, the final result for the heating temperature distribution $\Delta T(t, r)$ $\equiv T(t, \vec{r})-T_{0}$ in the Cartesian coordinates can be presented as follows:

$$
\begin{aligned}
\Delta T(t, \vec{r}) & =\int_{-\infty}^{+\infty} \frac{d \lambda_{x} d \lambda_{y}}{(2 \pi)^{2}} \int_{0}^{+\infty} \frac{2 d \lambda_{z}}{\pi} \Theta\left(\lambda_{x}, \lambda_{y}, \lambda_{z}, t\right) \Phi\left(\lambda_{x}, \lambda_{y}\right) \Psi\left(\lambda_{r}, \lambda_{z}\right) \exp \left[i\left(x \lambda_{x}+y \lambda_{y}\right)\right] Z_{\lambda_{z}}(z), \\
& +\int_{-\infty}^{+\infty} \frac{d \lambda_{x} d \lambda_{y}}{(2 \pi)^{2}} \sum_{n=1}^{N\left(\lambda_{r}\right)} \Theta_{n}\left(\lambda_{x}, \lambda_{y}, t\right) \Phi\left(\lambda_{x}, \lambda_{y}\right) \Psi_{n}\left(\lambda_{r}\right) \exp \left[i\left(x \lambda_{x}+y \lambda_{y}\right)\right] Z_{\chi_{n}}(z)
\end{aligned}
$$

where $\lambda_{r}=\sqrt{\lambda_{x}^{2}+\lambda_{y}^{2}}$, and functions $Z_{\lambda_{z}}(z)$ and $Z_{\chi_{n}}(z)$ are the boundary problem eigenfunctions corresponding to continuous and discrete spectrum of the eigenvalues, $\lambda_{z}$ and $\chi_{n}$, respectively. In expression (6), the different functions are defined by the following integrals:

$$
\begin{gathered}
\Phi\left(\lambda_{x}, \lambda_{y}\right)=\int_{-\infty}^{+\infty} \int_{-\infty}^{+\infty} \exp \left[-i\left(x \lambda_{x}+y \lambda_{y}\right)\right] f_{X Y}(x, y) d x d y, \\
\Theta\left(\lambda_{x}, \lambda_{y}, \lambda_{z}, t\right)=\exp \left(-i v \lambda_{x} t\right) \int_{0}^{t} f_{T}\left(t^{\prime}\right) \exp \left\{\left[D_{s}\left(\lambda_{r}^{2}+\lambda_{z}^{2}\right)-i v \lambda_{x}\right]\left(t^{\prime}-t\right)\right\} d t^{\prime}, \\
\Theta_{n}\left(\lambda_{x}, \lambda_{y}, t\right)=\exp \left(-i v \lambda_{x} t\right) \int_{0}^{t} f_{T}\left(t^{\prime}\right) \exp \left\{\left[D_{s}\left(\lambda_{r}^{2}-\chi_{n}^{2}\right)-i v \lambda_{x}\right]\left(t^{\prime}-t\right)\right\} d t^{\prime}, \\
\Psi\left(\lambda_{r}, \lambda_{z}\right)=\frac{1}{c_{s}}\left\{\int_{0}^{d} d z f_{Z}^{l}(z) Z_{\lambda_{z}}(z)+\int_{d}^{+\infty} d z f_{Z}^{s}(z) Z_{\lambda_{z}}(z)\right\}, \\
\Psi_{n}\left(\lambda_{r}\right)=\frac{1}{c_{s}}\left\{\int_{0}^{d} d z f_{Z}^{l}(z) Z_{\chi_{n}}(z)+\int_{d}^{+\infty} d z f_{Z}^{s}(z) Z_{\chi_{n}}(z)\right\} .
\end{gathered}
$$

Here and after, the designations $D_{l}=\sqrt{k_{l} / c_{l}}$ and $D_{s}=\sqrt{k_{s} / c_{s}}$ for the thermal diffusivity coefficients of the boundary layer and the substrate, respectively, are used.

For many particular cases important for applications, the integrals in expressions (7a-e) can be taken analytically. For example, function $\Phi\left(\lambda_{x}, \lambda_{y}\right)$, that is defined by the spatial distribution $f_{X Y}(x, y)$ of the laser intensity, can be simplified in the case of the Gaussian distribution, when $f_{X Y}(x, y)=\exp \left(-\left(x^{2}+y^{2}\right) / r_{0}^{2}\right)$, and also for the homogeneous distributions restricted either by a circle of radius $r_{0}$ or by a square with square side $2 r_{0}$ :

$$
\Phi\left(\lambda_{x}, \lambda_{y}\right)=\left\{\begin{array}{rl}
\pi r_{0}^{2} \exp \left(-\frac{\lambda_{r}^{2} r_{0}^{2}}{4}\right) & \text { - for the Gaussian distribution } \\
\frac{2 \pi r_{0}}{\lambda_{r}} J_{1}\left(r_{0} \lambda_{r}\right) & \text { - for the circle-restricted homogeneous distribution } \\
\frac{4 \sin \left(\lambda_{x} r_{0}\right) \sin \left(\lambda_{y} r_{0}\right)}{\lambda_{x} \lambda_{y}} & \text { - for the square-restricted homogeneous distribution }
\end{array} .\right.
$$

Similarly, functions $\Theta\left(\lambda_{x}, \lambda_{y}, \lambda_{z}, t\right)$ and $\Theta_{n}\left(\lambda_{x}, \lambda_{y}, t\right)$ (they are defined by the time dependence of the laser intensity) can be simplified for continuous laser radiation, or for the pulsed laser radiation with rectangular pulses (and also with the pulses of some other specific forms). For continuous laser radiation with $f_{T}(t)=0$ at $t<0$ and $f_{T}(t)=1$ at $\left.t\right\rangle$ 0 , these functions are reduced to the following:

$$
\Theta\left(\lambda_{x}, \lambda_{y}, \lambda_{z}, t\right)=\frac{\exp \left(-i v \lambda_{x} t\right)\left\{1-\exp \left(-\Lambda_{z} t\right)\right\}}{\Lambda_{z}}, \Theta_{n}\left(\lambda_{x}, \lambda_{y}, t\right)=\frac{\exp \left(-i v \lambda_{x} t\right)\left\{1-\exp \left(-\Lambda_{n} t\right)\right\}}{\Lambda_{n}},
$$

where $\Lambda_{z}=D_{s}\left(\lambda_{r}^{2}+\lambda_{z}^{2}\right)-i v \lambda_{x}$ and $\Lambda_{n}=D_{s}\left(\lambda_{r}^{2}-\chi_{n}^{2}\right)-i v \lambda_{x}$. For repeating rectangular laser pulses with duration $\tau_{p}$ and repetition rate $v_{L}$ (when $f_{T}(t)=1$ at $m / v_{L}<t<\tau_{p}+m / v_{L}, m=0,1,2, \ldots$, and otherwise $\left.f_{T}(t)=0\right)$ these functions are expressed as follows: 


$$
\begin{aligned}
& \Theta\left(\lambda_{x}, \lambda_{y}, \lambda_{z}, t\right)=\exp \left(-i \lambda_{x} v t\right) \\
& \times\left\{\begin{array}{lrr}
\frac{\left(e^{\Lambda_{z} m\left(\tau_{p}+\tau_{b p}\right)}-1\right)\left(e^{\Lambda_{z} \tau_{p}}-1\right) e^{-\Lambda_{z} t}}{\left(e^{\Lambda_{z}\left(\tau_{p}+\tau_{b p}\right)}-1\right) \Lambda_{z}}+\frac{1-e^{\Lambda_{z}\left(m\left(\tau_{p}+\tau_{b p}\right)-t\right)}}{\Lambda_{z}} & \text { for } & m\left(\tau_{p}+\tau_{b p}\right)<t<m\left(\tau_{p}+\tau_{b p}\right)+\tau_{p}, \\
\frac{\left(e^{\Lambda_{z}(m+1)\left(\tau_{p}+\tau_{b p}\right)}-1\right)\left(e^{\Lambda_{z} \tau_{p}}-1\right) e^{-\Lambda_{z} t}}{\left(e^{\Lambda_{z}\left(\tau_{p}+\tau_{b p}\right)}-1\right) \Lambda_{z}} & \text { for } & m\left(\tau_{p}+\tau_{b p}\right)+\tau_{p}<t<(m+1)\left(\tau_{p}+\tau_{b p}\right),
\end{array}\right. \\
& \Theta_{n}\left(\lambda_{x}, \lambda_{y}, t\right)=\exp \left(-i \lambda_{x} v t\right) \\
& \times\left\{\begin{array}{lcc}
\frac{\left(e^{\Lambda_{n} m\left(\tau_{p}+\tau_{b p}\right)}-1\right)\left(e^{\Lambda_{n} \tau_{p}}-1\right) e^{-\Lambda_{n} t}}{\left(e^{\Lambda_{n}\left(\tau_{p}+\tau_{b p}\right)}-1\right) \Lambda_{n}}+\frac{1-e^{\Lambda_{n}\left(m\left(\tau_{p}+\tau_{b p}\right)-t\right)}}{\Lambda_{n}} & \text { for } & m\left(\tau_{p}+\tau_{b p}\right)<t<m\left(\tau_{p}+\tau_{b p}\right)+\tau_{p}, \\
\frac{\left(e^{\Lambda_{n}(m+1)\left(\tau_{p}+\tau_{b p}\right)}-1\right)\left(e^{\Lambda_{n} \tau_{p}}-1\right) e^{-\Lambda_{n} t}}{\left(e^{\Lambda_{n}\left(\tau_{p}+\tau_{b p}\right)}-1\right) \Lambda_{n}} & \text { for } & m\left(\tau_{p}+\tau_{b p}\right)+\tau_{p}<t<(m+1)\left(\tau_{p}+\tau_{b p}\right),
\end{array}\right.
\end{aligned}
$$

with $\tau_{b p}$ the time between two consecutive pulses, so $\tau_{b p}=1 / \nu_{L}-\tau_{p}$. The simple expressions for these functions can be also obtained if the laser is switched off at the moment $t=T$, giving the description of the relaxation regime of surface cooling.

Let us discuss functions $\Psi\left(\lambda_{r}, \lambda_{z}\right)$ and $\Psi_{n}\left(\lambda_{r}\right)$ defined by expressions (7d) and (7e), respectively. One should know eigenfunctions of our boundary problem, $Z_{\lambda_{z}}(z)$ and $Z_{\chi_{n}}(z)$, which are involved in the expressions (7d-e), and also in expression (6) for the heating temperature. For arbitrary possible eigenvalue $\lambda_{z}$, the eigenfunction $Z_{\lambda_{z}}(z)$ should satisfy the equations:

with the boundary conditions:

$$
\begin{array}{r}
Z_{\lambda_{z}}^{\prime \prime}=-\left[\left(\lambda_{r}^{2}+\lambda_{z}^{2}\right) D_{s} / D_{l}-\lambda_{r}^{2}\right] Z_{\lambda_{z}} \quad(0<z<d), \\
Z_{\lambda_{z}}^{\prime \prime}=-\lambda_{z}^{2} Z_{\lambda_{z}}(z>d),
\end{array}
$$

$$
k_{l} Z_{\lambda_{z}}^{\prime}(d-0)=k_{s} Z_{\lambda_{z}}^{\prime}(d+0), \quad Z_{\lambda_{z}}(d-0)-Z_{\lambda_{z}}(d+0)=-\frac{k_{l}}{h} Z_{\lambda_{z}}^{\prime}(d-0), \quad Z_{\lambda_{z}}^{\prime}(0)=0,
$$

the latter is written for $\mu=0$, i.e., neglecting the convection heat transfer on the external surface boundary at $z=0$. For the heating problem with a deposited layer on the surface, the eigenvalue spectrum generally contains both the continuous part and the discrete part. The continuous part of the spectrum corresponds to the real $\lambda_{z}$ values. In this case, the solution of equations (11a) and (11b), together with the boundary conditions (12), is as follows:

$$
Z_{\lambda_{z}}(z)=\frac{\cos \lambda z}{\sqrt{B^{2}+C^{2}}} \quad(0<z<d), \quad Z_{\lambda_{z}}(z)=\frac{B \cos \lambda_{z} z+C \sin \lambda_{z} z}{\sqrt{B^{2}+C^{2}}} \quad(z>d),
$$

where $\lambda=\sqrt{\left(\lambda_{r}^{2}+\lambda_{z}^{2}\right) D_{s} / D_{l}-\lambda_{r}^{2}}$, and

$$
\begin{aligned}
& B=\cos \lambda_{z} d\left(\cos \lambda d-\frac{k_{l} \lambda}{h} \sin \lambda d\right)+\frac{k_{l} \lambda}{k_{s} \lambda_{z}} \sin \lambda_{z} d \sin \lambda d, \\
& C=\sin \lambda_{z} d\left(\cos \lambda d-\frac{k_{l} \lambda}{h} \sin \lambda d\right)-\frac{k_{l} \lambda}{k_{s} \lambda_{z}} \cos \lambda_{z} d \sin \lambda d .
\end{aligned}
$$

In the limit of $h \rightarrow 0$, that is for completely imperfect heat contact between the layer and the substrate, the continuous eigenfunctions generally vanish in $0<z<d$ range, i.e., in the range of the layer, but only except for the case of $\sin (\lambda d)=0$, so in this limit the continuous spectrum is continuously transformed to the discrete one solely presented in the case of $h=0$.

For the discrete set of eigenvalues, with pure imaginary values of $\lambda_{z}$, we introduce the designation $\lambda_{z}=i \chi(\chi>$ 0 ). The set of discrete eigenvalues is denoted as $\chi_{n}$ with $n=1,2, \ldots$. Then, the (normalized) eigenfunctions $Z_{\chi_{n}}(z)$ of the discrete spectrum have the form:

$$
\begin{aligned}
Z_{\chi_{n}}(z)=a_{n} \cos \left(\lambda_{n} z\right) & (0<z<d), \\
Z_{\chi_{n}}(z)=a_{n}\left(\cos \lambda_{n} d-\frac{k_{l} \lambda_{n}}{h} \sin \lambda_{n} d\right) \exp \left[-\chi_{n}(z-d)\right] & (z>d),
\end{aligned}
$$

where $a_{n}$ is the eigenfunction normalization constant, $\lambda_{n}=\sqrt{\left(\lambda_{r}^{2}-\chi_{n}^{2}\right) D_{s} / D_{l}-\lambda_{r}^{2}}$, and the $\chi_{n}$-value should satisfy the equation:

$$
\lambda_{n} \tan \left(\lambda_{n} d\right)=\frac{h k_{s} \chi_{n}}{k_{l}\left(h+k_{s} \chi_{n}\right)} .
$$

The normalization constant $a_{n}$ for an eigenfunction $Z_{\chi_{n}}(z)$ of the discrete spectrum is determined by the relation:

$$
a_{n}^{-2}=\frac{c_{l}}{c_{s}}\left\{\frac{d}{2}+\frac{\sin \left(2 d \lambda_{n}\right)}{4 \lambda_{n}}\right\}+\frac{\left[\cos \left(d \lambda_{n}\right)-\left(k_{l} \lambda_{n} / h\right) \sin \left(d \lambda_{n}\right)\right]^{2}}{2 \chi_{n}} .
$$


While analyzing the equation (16) for the discrete spectrum of eigenvalues, the following conclusions can be made. For $D_{s} / D_{l} \leq 1$, equation (16) for each value of $\lambda_{r}$ has no solutions. On the contrary, for $D_{s} / D_{l}>1$, the discrete spectrum is always present (except only for 1D case, where we should put anywhere $\lambda_{r}=0$ ). For each finite value of $\lambda_{r}$, the solutions belong to $0<\chi_{n}<\lambda_{r} \sqrt{1-D_{l} / D_{s}}$ range, where quantity $\lambda_{n}$ is real, and the number of solutions increases with increasing the value of $\lambda_{r}$. If $N\left(\lambda_{r}\right) \geq 1$ denotes the total finite number of solutions of equation (16) for a given value of $\lambda_{r}$, then $\chi_{n}\left(\lambda_{r}\right)$ denotes the discrete eigenvalue for $1 \leq n \leq N\left(\lambda_{r}\right)$.

Now we have all the information to determine the functions $\Psi\left(\lambda_{r}, \lambda_{z}\right)$ and $\Psi_{n}\left(\lambda_{r}\right)$ defined by expressions (7d) and (7e), respectively. By carrying out analytically the integration over $z$ with the expressions (4a-b), (13) and (15a-b), we obtain that:

$$
\begin{gathered}
\Psi\left(\lambda_{r}, \lambda_{z}\right)=\frac{\left(\tilde{a}-\tilde{b} e^{-2 d \alpha_{l}}\right) \alpha_{l}+e^{-d \alpha_{l}}\left[(\tilde{a}+\tilde{b}) \lambda \sin \lambda d-(\tilde{a}-\tilde{b}) \alpha_{l} \cos \lambda d\right]}{c_{s}\left(\lambda^{2}+\alpha_{l}^{2}\right) \sqrt{B^{2}+C^{2}}} \\
+\frac{\tilde{c} e^{-d \alpha_{l}}\left\{\left(C \alpha_{s}-B \lambda_{z}\right) \sin \lambda_{z} d+\left(B \alpha_{s}+C \lambda_{z}\right) \cos \lambda_{z} d\right\}}{c_{s}\left(\lambda_{z}^{2}+\alpha_{s}^{2}\right) \sqrt{B^{2}+C^{2}}}, \\
\Psi_{n}\left(\lambda_{r}\right)=\frac{a_{n}\left\{\left(\tilde{a}-\tilde{b} e^{-2 d \alpha_{l}}\right) \alpha_{l}+e^{-d \alpha_{l}}\left[(\tilde{a}+\tilde{b}) \lambda_{n} \sin \lambda_{n} d-(\tilde{a}-\tilde{b}) \alpha_{l} \cos \lambda_{n} d\right]\right\}}{c_{s}\left(\lambda_{n}^{2}+\alpha_{l}^{2}\right)} \\
+\frac{a_{n} \tilde{c} e^{-d \alpha_{l}}\left\{\cos \lambda_{n} d-\left(k_{l} \lambda_{n} / h\right) \sin \lambda_{n} d\right\}}{c_{s}\left(\chi_{n}+\alpha_{s}\right)} .
\end{gathered}
$$

Here, the following designations are introduced:

$$
\tilde{a}=I_{0} \frac{\omega \varepsilon_{l}^{\prime \prime}}{4 c}\left|\tilde{C}_{s}\right|^{2}\left|1+\sqrt{\frac{\varepsilon_{s}}{\varepsilon_{l}}}\right|^{2}, \quad \tilde{b}=I_{0} \frac{\omega \varepsilon_{l}^{\prime \prime}}{4 c}\left|\tilde{C}_{s}\right|^{2}\left|1-\sqrt{\frac{\varepsilon_{s}}{\varepsilon_{l}}}\right|^{2}, \quad \tilde{c}=I_{0} \frac{\omega}{c} \varepsilon_{s}^{\prime \prime}\left|\tilde{C}_{s}\right|^{2} .
$$

The obtained formulae finalize the description of the analytical solution of laser heating of a complex surface with an intermediate heat contact between the boundary layer and the substrate in a rectangular geometry, for a general case of a moving laser beam.

In conclusion, we also present the simplified version of this solution in a cylindrical geometry, which can be successfully used for immobile laser beam (when $v=0$ ) irradiating the given area on the surface, if the laser spot has a circular symmetry. In a cylindrical geometry, the heating temperature of the initially unheated medium being at $t<0$ in equilibrium with the environment is described by the expression:

$$
\begin{aligned}
\Delta T(t, r, z) & =\int_{0}^{+\infty} d \lambda_{r} \int_{0}^{+\infty} \frac{2 d \lambda_{z}}{\pi} \Theta\left(\lambda_{r}, \lambda_{z}, t\right) \Phi\left(\lambda_{r}\right) \Psi\left(\lambda_{r}, \lambda_{z}\right) J_{0}\left(r \lambda_{r}\right) Z_{\lambda_{z}}(z) \\
& +\int_{0}^{+\infty} d \lambda_{r} \sum_{n=1}^{N\left(\lambda_{r}\right)} \Theta_{n}\left(\lambda_{r}, t\right) \Phi\left(\lambda_{r}\right) \Psi_{n}\left(\lambda_{r}\right) J_{0}\left(r \lambda_{r}\right) Z_{\chi_{n}}(z) .
\end{aligned}
$$

Here, we should also put the velocity $v=0$ in expressions (7b-c), (9) and (10a-b) for $\Theta$-functions. The more simplifications can be made in the 1D case. This case can be relevant for a wide laser spot, at specific set of the thermophysical and optical parameters of the media, at least at sufficiently short time of laser heating. In this case, one numerical integration over $\lambda_{z}$ is only kept, providing fast calculations of the laser heating of the surface.

To obtain the final quantitative results by analytical approach, the sizable numerical calculations should be still made according to the obtained expressions with multiple integrals and multi-scale functions. It is significant that in such calculations, the limitations on total duration of the laser heating are much lower. The calculations can be made for much longer time intervals of laser heating than it can be made by direct numerical simulations with the time-consuming finitedifference methods. The obtained analytical solution of laser heating of complex surfaces was implemented in the computer code for numerical simulations. The calculations were made for different cases of the laser heating of the surfaces without and with a deposited layer. The extensive simulations of laser heating were applied to graphite surface with a deposited layer.

The different regimes of the heating by a pulsed laser radiation (with $5 \mathrm{~ns}-50 \mathrm{~ms}$ pulse duration and with the pulse repetition rate of $20-10000 \mathrm{~Hz}$ ) as well as by a continuous laser were considered. The laser beam can be immobile or moving along the surface during the heating. The various temporal and spatial distributions (either the Gaussian or homogeneous in the different beam geometries) of the laser radiation acting on the surface were taken into account. The heating temperature was calculated as a function of time for different points located both just on the surface and deep in the interior of the surface medium. Two limiting cases of the heat contact between the deposited micrometric layer and the substrate were considered. The main attention was paid to the 3D "analytical" model with the mean constant optical and thermo-physical parameters, which gives possibility to trace the heating temperature on large temporal scales. For validation of this approach, the temperature dependences of the thermo-physical properties (specific heat, thermal conductivity, etc.) of the surface substances were taken into account in direct numerical solution of the heat equations. The laser heating simulations were made for the surface of the technical graphite (both without and with the friable deposited layer), for the continuous and pulsed laser radiation.

The density of both the substrate and the layer was taken as $\rho=(1-p) \rho_{0}$, where $\rho_{0}=2240 \mathrm{~kg} \mathrm{~m}^{-3}$ is the density of monocrystal graphite and $p$ is the respective porosity. For technical graphite, it was taken as $p=0.25$, and for a thick 
co-deposited layer, it was found to be two times higher (in this case, it should be generally considered as an adjustable parameter). The thermal capacity (per unit of volume) was taken as $C_{p}=\rho C_{0}$, where $C_{0}=1500 \mathrm{~J} \mathrm{~kg}^{-1} \mathrm{~K}^{-1}$ is the mean reference value of the mass thermal capacity of dense crystal graphite (in the range of temperatures that we consider). For technical graphite, the constant thermal conductivity was taken as $k=100 \mathrm{~W} \mathrm{~m}^{-1} \mathrm{~K}^{-1}$. For a deposited layer, it was found to be three times lower (the thermal conductivity should also be considered as an adjustable parameter). The graphite with the chosen parameters has thermal diffusivity $D=k / C_{p} \approx 4 \times 10^{-5} \mathrm{~m}^{2} \mathrm{~s}^{-1}$. The numerical model for laser heating can determine the surface temperature as a function of time, in-depth position, interaction parameters, and surface properties. It was applied to graphite with a deposited layer of thickness $d=1 \mu \mathrm{m} \div 1 \mathrm{~mm}$ and for the laser pulses in $\mathrm{ns} \div \mathrm{ms}$ range with the pulse repetition rate up to $10 \mathrm{kHz}$.

\section{Phase shifts calculations}

\subsection{Basic equations and solutions for the stationary periodical laser heating}

The equations for time-dependent heating temperature $\Delta T(t, z, r) \equiv T(t, z, r)-T_{0}$ of a complex surface with a layer deposited on the substrate ( $T_{0}$ is the ambient temperature) can be written as they are presented in Sec. 2.1. In the stationary regime of laser heating, for the repetition rate laser with the repetition rate frequency $v_{L}$, the time-dependent laser intensity $I(t)$ (which is not obligatory of a simple sinusoidal form) can be expressed as the Fourier series:

$$
\begin{aligned}
& I(t)=I_{0}+\sum_{n=1}^{\infty}\left(a_{n} \sin 2 \pi n v_{L} t+b_{n} \cos 2 \pi n v_{L} t\right)=I_{0}+\sum_{n=1}^{\infty} \sqrt{a_{n}^{2}+b_{n}^{2}} \sin \left(2 \pi n v_{L} t+\varphi_{L P}^{(n)}\right) \\
& =\operatorname{Re}\left\{I_{0}+i \sum_{n=1}^{\infty} \sqrt{a_{n}^{2}+b_{n}^{2}} e^{-i\left(2 \pi n v_{L} t+\varphi_{L P}^{(n)}\right)}\right\} \equiv \operatorname{Re} \sum_{n=0}^{\infty} I_{n} e^{-i 2 \pi n v_{L} t},
\end{aligned}
$$

where $I_{0}$ is the mean laser intensity, and $\varphi_{L P}^{(n)}=\tan ^{-1}\left(b_{n} / a_{n}\right)$ and $I_{n}=i \sqrt{a_{n}^{2}+b_{n}^{2}} e^{-i \varphi_{L P}^{(n)}}$ are, respectively, the laser pulse phase and the laser complex intensity corresponding to the $n^{\text {th }}$ - order Fourier harmonic of the pulse repetition rate laser frequency $v_{L}$. The mean laser intensity over the laser period should be normalised by the mean laser power $P$ as $I_{0}=v_{L} \int_{t^{*}}^{t^{*}+1 / v_{L}} I(t) d t=P /\left(\pi r_{0}^{2}\right)$, and the coefficients $a_{n}$ and $b_{n}(n>0)$ can be determined either from the experimental or from the simulated time-dependent laser intensity from the relations:

$$
\mathrm{b}_{\mathrm{n}}=2 v_{\mathrm{L}} \int_{\mathrm{t}^{*}}^{\mathrm{t}^{*}+1 / v_{\mathrm{L}}} \mathrm{I}(\mathrm{t}) \cos \left(2 \pi \mathrm{n} v_{\mathrm{L}} \mathrm{t}\right) \mathrm{dt}, \quad \mathrm{a}_{\mathrm{n}}=2 v_{\mathrm{L}} \int_{\mathrm{t}^{*}}^{\mathrm{t}^{*}+1 / v_{\mathrm{L}}} \mathrm{I}(\mathrm{t}) \sin \left(2 \pi \mathrm{n} v_{\mathrm{L}} \mathrm{t}\right) \mathrm{dt}
$$

so that

$$
\tan \varphi_{\mathrm{LP}}^{(\mathrm{n})}=\frac{\mathrm{b}_{\mathrm{n}}}{\mathrm{a}_{\mathrm{n}}}=\int_{\mathrm{t}^{*}}^{\mathrm{t}^{*}+1 / \nu_{\mathrm{L}}} \mathrm{I}(\mathrm{t}) \cos \left(2 \pi \mathrm{n} v_{\mathrm{L}} \mathrm{t}\right) \mathrm{dt} \int_{\mathrm{t}^{*}+1 / \nu_{\mathrm{L}}}^{*} \mathrm{I}(\mathrm{t}) \sin \left(2 \pi \mathrm{n} v_{\mathrm{L}} \mathrm{t}\right) \mathrm{dt}
$$

where $t^{\star}$ is an arbitrary time moment within the stationary regime. For example, for the rectangular laser pulses starting at $\mathrm{t}=0$ with the duration of $0.5 v_{L}^{-1}$, that is, one half fraction of the laser repetition period, $a_{n}=\left(2 I_{0} /(\pi n)\right)(1-\cos (\pi n))$ and $b_{n}=0$, so that $\varphi_{L P}^{(n)}=0$.

The thermal response of the sample can also be expressed as the Fourier series:

$$
\begin{aligned}
& \Delta T(t, z, r)=\Delta T_{0}(z, r)+\sum_{n=1}^{\infty}\left(A_{n}(z, r) \sin 2 \pi n v_{L} t+B_{n}(z, r) \cos 2 \pi n v_{L} t\right) \\
& =\Delta T_{0}(z, r)+\sum_{n=1}^{\infty} \sqrt{A_{n}^{2}+B_{n}^{2}} \sin \left(2 \pi n v_{L} t+\varphi_{L H}^{(n)}\right)=\operatorname{Re}\left\{\Delta T_{0}(z, r)+i \sum_{n=1}^{\infty} \sqrt{A_{n}^{2}+B_{n}^{2}} e^{-i\left(2 \pi n v_{L} t+\varphi_{L H}^{(n)}\right)}\right\}, \\
& \equiv \operatorname{Re} \sum_{n=0}^{\infty} \Delta T_{n}(z, r) e^{-i 2 \pi n v_{L} t}
\end{aligned}
$$

where $\Delta T_{0}(z, r)$ is the mean steady-state heating temperature, and $\varphi_{L H}^{(n)}(z, r)=\tan ^{-1}\left(B_{n} / A_{n}\right)$ and $\Delta T_{n}=i \sqrt{A_{n}^{2}+B_{n}^{2}} e^{-i \varphi_{L H}^{(n)}}$ are, respectively, the temperature phase and the complex temperature amplitude corresponding to the $n^{\text {th }}$-order harmonic of the pulse repetition rate frequency $v_{L}$. If the temperature trace is known either from the experiments or calculations, the heating temperature phase can be found from the expression, similar to the expression (23) for the laser pulse:

$$
\tan \varphi_{L H}^{(n)}(z, r)=\frac{B_{n}(z, r)}{A_{n}(z, r)}=\int_{t^{*}}^{t^{*}+1 / v_{L}} \Delta T(t, z, r) \cos \left(2 \pi n v_{L} t\right) d t \int_{t^{*}}^{t^{*}+1 / v_{L}} \Delta T(t, z, r) \sin \left(2 \pi n v_{L} t\right) d t .
$$


The temperature is measured in the laser spot centre on the surface ( $z=0$ and $r=0)$. Both the expressions (23) and (25) should be used for the phase shifts calculations of experimentally measured temperature traces with respect to the laser pulse phase. The mean heating temperature increasing rate (mean temperature derivative) should be removed if the measurements are made before the stationary regime of laser heating. The theoretical phase shifts can be derived from the calculated temperature traces obtained with the developed numerical model. This approach is very timeconsuming, especially in 3-D regime. The method of the direct phase shift calculations presented below seems to be more attractive.

\subsection{Method of the direct phase shift calculations}

For the temperature Fourier amplitudes $\Delta T_{n}(z, r)$, the following equations can be obtained:

$$
\begin{array}{rlrl}
-i 2 \pi n v_{L} \rho_{l} C_{l} \Delta T_{n} & =k_{l}\left(\frac{\partial^{2} \Delta T_{n}}{\partial z^{2}}+\frac{\partial^{2} \Delta T_{n}}{\partial r^{2}}+\frac{1}{r} \frac{\partial \Delta T_{n}}{\partial r}\right)+\alpha_{l}\left(1-R_{l}\right) I_{n} \exp \left(-\alpha_{l} z\right) f_{L}(r) & & (0<z<d) \\
& -i 2 \pi n v_{L} \rho_{s} C_{s} \Delta T_{n}=k_{s}\left(\frac{\partial^{2} \Delta T_{n}}{\partial z^{2}}+\frac{\partial^{2} \Delta T_{n}}{\partial r^{2}}+\frac{1}{r} \frac{\partial \Delta T_{n}}{\partial r}\right) . & (z \geq d)
\end{array}
$$

The general solution of these equations can be sought as:

$$
\begin{array}{cc}
\Delta T_{n}(z, r)=\int_{0}^{+\infty}\left(F_{1 n}(\xi) e^{-\kappa_{1 n} z}+F_{2 n}(\xi) e^{k_{1 n} z}\right) J_{0}(\xi r) d \xi+\frac{\alpha_{l}\left(1-R_{l}\right) I_{n} e^{-\alpha_{l} z}}{k_{l}} f_{n}(r), & (0<z<d) \\
\Delta T_{n}(z, r)=\int_{0}^{+\infty} F_{3 n}(\xi) e^{-\kappa_{2 n} z} J_{0}(\xi r) d \xi, & (z \geq d)
\end{array}
$$

where $\kappa_{1 n}=\sqrt{\xi^{2}-2 \pi i n v_{L} \rho_{l} C_{l} / k_{l}}, \quad \kappa_{2 n}=\sqrt{\xi^{2}-2 \pi i n v_{L} \rho_{s} C_{s} / k_{s}}\left(\operatorname{Re} \kappa_{1 n}>0\right.$ and $\left.\operatorname{Re} \kappa_{2 n}>0\right)$, and $f_{n}(r)$ is a particular solution of the inhomogeneous equation

$$
\frac{\partial^{2} f_{n}}{\partial r^{2}}+\frac{1}{r} \frac{\partial f_{n}}{\partial r}+\left(\alpha_{l}^{2}+\frac{i 2 \pi n v_{L} \rho_{l} C_{l}}{k_{l}}\right) f_{n}+f_{L}(r)=0
$$

which we will seek as $f_{n}(r)=\int_{0}^{+\infty} F_{n}(\xi) J_{0}(\xi r) d \xi$. Then,

with

$$
F_{n}(\xi)=\frac{\Phi(\xi)}{\xi^{2}-\alpha_{l}^{2}-2 \pi i n v_{L} \rho_{l} C_{l} / k_{l}}
$$

$$
\Phi(\xi)=\int_{0}^{+\infty} \xi r f_{L}(r) J_{0}(\xi r) d r=\left\{\begin{array}{l}
\frac{\xi r_{0}^{2}}{2} \exp \left(-\frac{\xi^{2} r_{0}^{2}}{4}\right) \text { - for the Gaussian distribution } \\
r_{0} J_{1}\left(\xi r_{0}\right) \quad \text { - for the flat }- \text { top distribution }
\end{array}\right.
$$

The initial condition $\Delta T(t=0, \vec{r}) \equiv T(t=0, \vec{r})-T_{0}=0$ was used in the expressions (27) for the stationary regime. Three boundary conditions $(2 \mathrm{a}),(2 \mathrm{~b})$ and $(2 \mathrm{c})$ (the latter with $\mu=0$ ) result in three linear relations between the functions $F_{1 n}(\xi), F_{2 n}(\xi)$ and $F_{3 n}(\xi)$ :

$$
\begin{gathered}
F_{1 n}-F_{2 n}=-\frac{\alpha_{l}^{2}\left(1-R_{l}\right) I_{n}}{k_{l} \kappa_{1 n}} F_{n}, \\
F_{1 n} e^{-\kappa_{1 n} d}-F_{2 n} e^{\kappa_{1 n} d}-\frac{k_{s} \kappa_{2 n}}{k_{l} \kappa_{1 n}} F_{3 n} e^{-\kappa_{2 n} d}=-\frac{\alpha_{l}^{2}\left(1-R_{l}\right) I_{n}}{k_{l} \kappa_{1 n}} e^{-\alpha_{l} d} F_{n}, \\
F_{1 n} e^{-\kappa_{1 n} d}+F_{2 n} e^{\kappa_{1 n} d}-\left(1+k_{s} \kappa_{2 n} / h\right) F_{3 n} e^{-\kappa_{2 n} d}=-\frac{\alpha_{l}\left(1-R_{l}\right) I_{n}}{k_{l}} e^{-\alpha_{l} d} F_{n} .
\end{gathered}
$$

The theoretical heating temperature corresponding to the one measured by the pyrometer can be found from the integral expression:

$$
\Delta T_{n}(z=0, r=0)=\int_{0}^{+\infty}\left(F_{1 n}(\xi)+F_{2 n}(\xi)+\frac{\alpha_{l}\left(1-R_{l}\right) I_{n}}{k_{l}} F_{n}(\xi)\right) d \xi \equiv \int_{0}^{+\infty}\left(2 F_{2 n}(\xi)+\frac{\alpha_{l}\left(1-R_{l}\right) I_{n}}{k_{l}}\left(1-\frac{\alpha_{l}}{\kappa_{1 n}}\right) F_{n}(\xi)\right) d \xi .
$$

The solution of the system of linear equations (31) for $F_{2 n}(\xi)$ is:

$$
F_{2 n}(\xi)=\frac{\alpha_{l}^{2}\left(1-R_{l}\right) I_{n} F_{n}(\xi)}{k_{l} \kappa_{1 n} D}\left\{\left(e^{-\alpha_{l} d}-e^{-\kappa_{1 n} d}\right)\left(1+\frac{k_{s} \kappa_{2 n}}{h}\right)+\left(e^{-\kappa_{1 n} d}-\frac{\kappa_{1 n}}{\alpha_{l}} e^{-\alpha_{l} d}\right) \frac{k_{s} \kappa_{2 n}}{k_{l} \kappa_{1 n}}\right\} e^{-\kappa_{1 n} d},
$$


with $D=\left(1+e^{-2 \kappa_{1 n} d}\right) \frac{k_{s} \kappa_{2 n}}{k_{l} \kappa_{1 n}}+\left(1-e^{-2 \kappa_{1 n} d}\right)\left(1+\frac{k_{s} \kappa_{2 n}}{h}\right)$. To obtain the final expression for the temperature calculations, a new integration variable $\tilde{\xi}=\xi r_{0}$ is introduced. The expression (32) can be rewritten as:

$$
\begin{aligned}
& \Delta T_{n}(z=0, r=0)=\frac{\alpha_{l}\left(1-R_{l}\right) I_{n}}{k_{l}} \int_{0}^{+\infty} \frac{\widetilde{\Phi}(\tilde{\xi})}{\widetilde{\xi}^{2} / r_{0}^{2}-\alpha_{l}^{2}-2 \pi i n v_{L} \rho_{l} C_{l} / k_{l}} \\
& \times\left[\frac{2 \alpha_{l} e^{-\kappa_{1 n} d}}{\kappa_{1 n} D}\left\{\left(e^{-\alpha_{l} d}-e^{-\kappa_{1 n} d}\right)\left(1+\frac{k_{s} \kappa_{2 n}}{h}\right)+\left(e^{-\kappa_{1 n} d}-\frac{\kappa_{1 n}}{\alpha_{l}} e^{-\alpha_{l} d}\right) \frac{k_{s} \kappa_{2 n}}{k_{l} \kappa_{1 n}}\right\}+\left(1-\frac{\alpha_{l}}{\kappa_{1 n}}\right)\right] d \tilde{\xi} .
\end{aligned}
$$

Here, $\widetilde{\Phi}(\tilde{\xi})=0.5 \tilde{\xi} \exp \left(-\widetilde{\xi}^{2} / 4\right)$ for the Gaussian laser beam and $\widetilde{\Phi}(\widetilde{\xi})=J_{1}(\widetilde{\xi})$ for the flat-top laser beam, and now $\kappa_{1 n}=\sqrt{\tilde{\xi}^{2} / r_{0}^{2}-2 \pi i n v_{L} \rho_{l} C_{l} / k_{l}}$ and $\kappa_{2 n}=\sqrt{\tilde{\xi}^{2} / r_{0}^{2}-2 \pi i n v_{L} \rho_{s} C_{s} / k_{s}}$. In the numerical calculations, the laser beam was assumed to be the Gaussian, which is preferable due to a fast vanishing of the function $\tilde{\Phi}(\tilde{\xi})$ with increasing $\tilde{\xi}$ (on the scale of $\tilde{\xi}<10$ ). It is not the case for the flat-top laser beam with a sharp edge. For the phase shift calculations, it is sufficient to consider only the particular Fourier contribution $\Delta T_{1}$ corresponding to $n=1$, that is, to the laser fundamental repetition rate frequency $v_{L}$. The phase shift can be found from the expression

$$
\Delta \varphi^{(1)} \equiv \varphi_{L H}^{(1)}-\varphi_{L P}^{(1)}=\operatorname{Re}\left\{i \ln \left(\Delta T_{1}(z=0, r=0) / \tilde{I}_{1}\right)\right\},
$$

while $\Delta T_{0}(z=0, r=0)$ and $\left|\Delta T_{1}(z=0, r=0)\right|$ can give the mean heating temperature and the temperature oscillations amplitude on the fundamental repetition rate frequency, respectively.

The phase shift does not depend on the temporal shape of the laser intensity if the optical and thermal properties of the layer and substrate do not depend on the temperature. Thus, if only phase shifts are required, it is not necessary to introduce the experimental temporal laser pulse shape in the simulations. The phase shift is determined for the Fourier component on the laser repetition rate frequency. In this case, the phase shifts calculations for 3-D laser heating may be performed much faster than with the very complex and time consuming method with the full temperature traces calculations.

The developed method of fast phase shifts calculations was applied to determine phase shift sensitivity. It was possible to calculate a phase shift as a function of $v_{L}$ and $h$ in a wide range of their values. Generally, the phase shift is a non-monotonous function of both $v_{L}$ and $h$. The phase shift also depends on $r_{0}$ (see Fig. 1). From these results, it was concluded that the influence of the laser repetition rate frequency on the phase shift is different for different heat contact coefficients in the range of $1<h\left[\mathrm{~kW} /\left(\mathrm{m}^{2} \mathrm{~K}\right)\right]<1000$ for $d=100 \mu \mathrm{m}$ and $0.1<h\left[\mathrm{~kW} /\left(\mathrm{m}^{2} \mathrm{~K}\right)\right]<10000$ for $d=10 \mu \mathrm{m}[11]$. In the latter case, the phase shift sensitivity is strongly decreasing if the heat contact coefficient is beyond both the high and low range limits. For $d=100 \mu \mathrm{m}$, the phase shift sensitivity is also decreasing with the important increase in the laser repetition rate frequency. In this case, the moderate repetition rate frequencies $\left(v_{L}<200 \mathrm{~Hz}\right)$ are preferable.
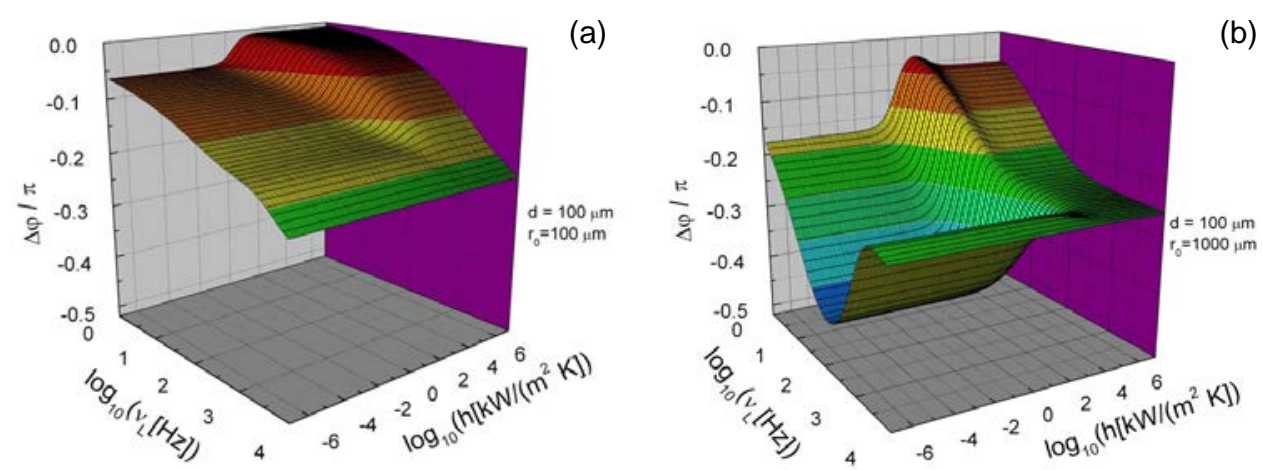

Fig. 1. The surface plots of the phase shift dependence on $v_{L}$ and $h$ for the TEXTOR graphite deposited layer on the TEXTOR graphite substrate, $d=100 \mu \mathrm{m}$. The laser beam radius is $r_{0}=100 \mu \mathrm{m}$ (a) and $r_{0}=1000 \mu \mathrm{m}(\mathrm{b})$.

\section{Experiments}

The developed theoretical models were applied to choose the relevant validation experiments. The setup is based on a Q-switched pulsed repetition rate Nd-YAG laser, an optical system for laser beam transportation, an optical focusing system, a generator to trigger laser pulses, a pyrometer system and PC software for pyrometer signal processing. The laser beam parameters were the following: $1064 \mathrm{~nm}$ wavelength, up to $200 \mathrm{~W}$ peak power; adjustable pulse duration $(0.5-500 \mathrm{~ms})$ and repetition rate $(1 \mathrm{~Hz} \div 10 \mathrm{kHz})$, beam divergence $\theta \approx 30 \mathrm{mrad}$; laser beam with a tophat intensity distribution and diameter $D \equiv 2 r_{0}=2 \mathrm{~mm}$ on the tested surface. The laser beam was transported by a $20-$ 
meter optical fiber (silica, $1 \mathrm{~mm}$ diameter, numerical aperture N.A.= 0.2). A pulse generator (Princeton, DG535) was used to control the applied laser pulses (number, pulse duration, repetition rate). A photodiode was used to register the laser power reflected from the surface to provide laser beam and surface reflectivity control.

The developed pyrometer system comprises two pyrometers (Kleiber KGAF 740-HS and Kleiber KGAF 274CLWL, $500-2600 \mathrm{~K}$ temperature range, $1.58-2.2 \mu \mathrm{m}$ spectral sensitivity range, time constant t99\% $=15 \mu \mathrm{s}$ ), an optical lens (focal length $F=61$ or $F=41 \mathrm{~mm}$ ) to image the heated zone of 500 or $700 \mu \mathrm{m}$ diameter onto the entrances of the pyrometer fiber ( $1.5 \mathrm{~m}$ length, $200 \mu \mathrm{m}$ diameter), and a computer system to process the input data. The pyrometers with the "direct" output provide the exit signal (in Volts) proportional to the photon flux. All the data were processed by the MATLAB software. The measurements were made on the tokamak graphite tiles with deposited layers (from TEXTOR or TORE SUPRA) and with W-layer ( $\approx 140 \mu \mathrm{m}$ tungsten thickness) deposited onto the CFC substrate by a plasma spray. Here, only results obtained with TEXTOR samples are presented.

\subsection{Laser active pyrometry method}

The laser acousto-optical modulator adjustments provided the laser pulses of a nearly rectangular shape for the applied repetition rates $v_{L}=2 \mathrm{~Hz}-1 \mathrm{kHz}$. Laser pulse duration was $\tau_{L} \cong\left(0.5 / v_{L}\right)$. Zones $\mathrm{B}, \mathrm{C}$, and $\mathrm{D}$ (Fig. 2$)$ with a carbon deposited layers were under laser heating with $v_{L}=2-1000 \mathrm{~Hz}$.

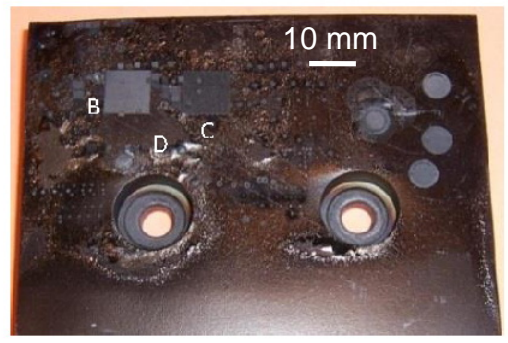

Fig. 2. Measured zones on TEXTOR sample.

The laser power was carefully adjusted to avoid the layer overheating which may affect the initial layer properties and results in undesirable modification in surface features. Thus, the applied laser power was $P=12 \mathrm{~W}$ for zone B (33 $\pm 13 \mu \mathrm{m}$ layer thickness), $P=12 \mathrm{~W}$ or $16 \mathrm{~W}$ for zone $\mathrm{C}(4 \pm 2 \mu \mathrm{m}$ layer thickness), and $P=8 \mathrm{~W}$ for zone $\mathrm{D}$ $(\approx 10 \mu \mathrm{m}$ layer thickness, detached), to provide the condition $\Delta T<1000 \mathrm{~K}$. The maximal heating temperature and modulation amplitude decrease with increasing of the laser repetition rate frequency. The experimental heating temperatures (Fig. 11-14) were determined for all the zones under study.

With the 3-D modelling, it was possible to calculate the heating temperature with a good temporal and spatial resolution. In our simulations, the graphite properties for the TEXTOR tile (substrate) were taken as in [2]: density $\rho_{\mathrm{s}}=$ $1680 \mathrm{~kg} / \mathrm{m}^{3}$, porosity $p_{s}=25 \%$, mass specific heat $C_{s}=1500 \mathrm{~J} /(\mathrm{kg} \mathrm{K})$, and thermal conductivity $k_{s}=60 \mathrm{~W} /(\mathrm{m} \mathrm{K})$. The fitting for the experimental/calculated temperatures for zones B, C, and D was made. For zones B, C, and D, respectively, Fig. 3, Fig. 4 and Fig. 5 present the best temperature fitting obtained with the adjusted carbon layer properties. The set of the layer parameters corresponding to the best fit of the experimental/simulation results was considered as the layer properties. Table 1 gives the obtained results for zones $B, C$ and $D$ and presents the adjusted mean carbon layer properties $\left(\rho_{1}, C_{1}, k_{1}, \alpha_{1}, R_{1}\right)$ and the thermal contact coefficient $h_{\text {alp }}$ determined by active laser pyrometry with $\approx 10 \%$ accuracy (for a given layer thickness $d$ ) for TEXTOR tile as a substrate.
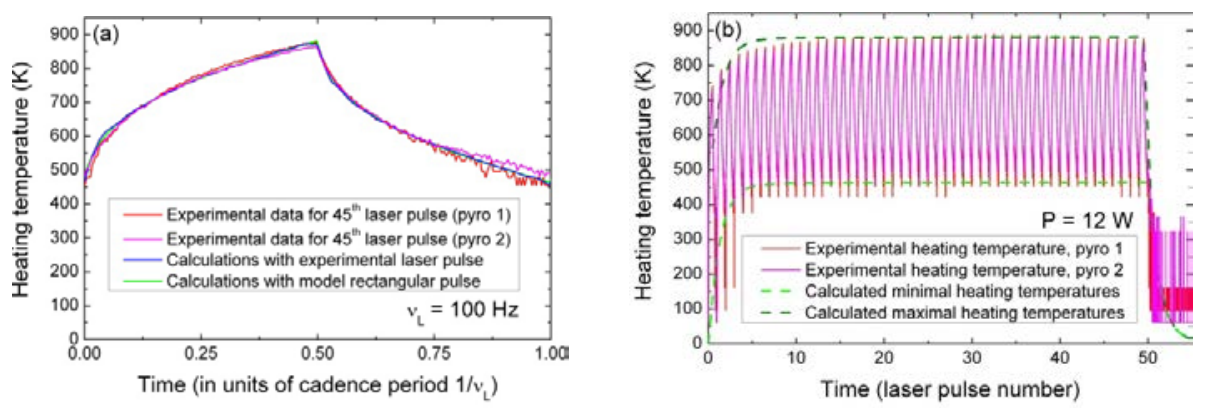

Fig. 3. Theoretical fit of the heating temperatures for zone $B\left(33 \pm 13 \mu \mathrm{m}\right.$ layer thickness). $P=12 W, v_{L}=100 \mathrm{~Hz}$. Panel (a) presents the results for the $45^{\text {th }}$ laser pulse in the saturation regime for mean temperature. Panel (b) presents the profiles of the minimal and maximal heating temperatures for fifty laser pulses on the whole temperature trace. 

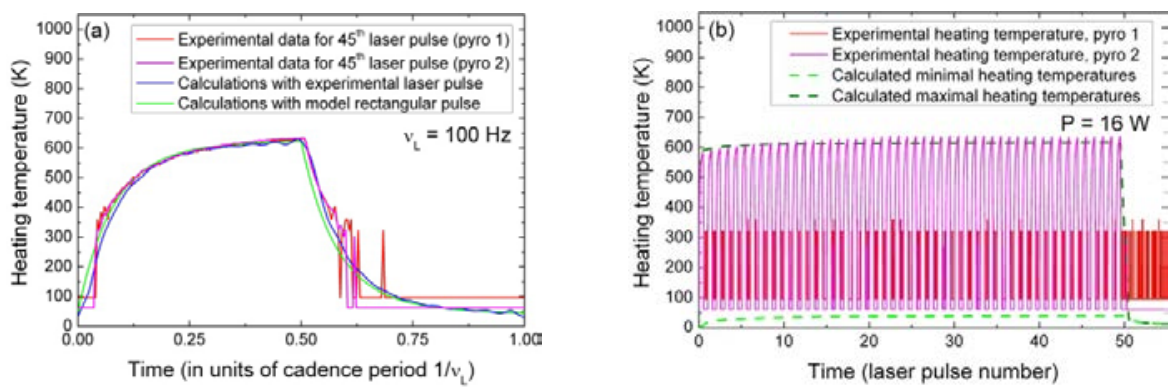

Fig. 4. Theoretical fit of the heating temperatures for zone $C\left(4 \pm 2 \mu \mathrm{m}\right.$ layer thickness). $P=16 \mathrm{~W}, v_{L}=100 \mathrm{~Hz}$. Panel (a) presents the results for the $45^{\text {th }}$ laser pulse in the saturation regime for mean temperature. Panel (b) presents the profiles of the minimal and maximal heating temperatures for fifty laser pulses on the whole temperature trace.
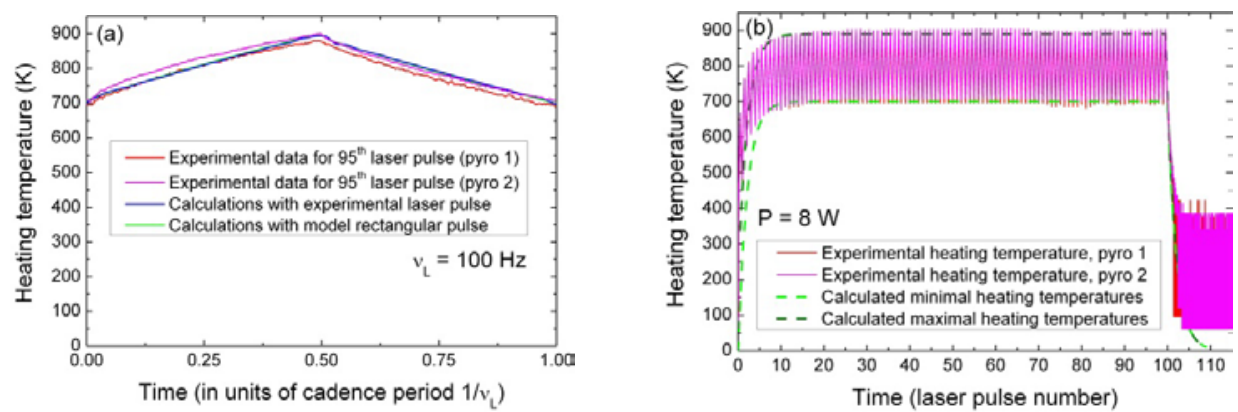

Fig. 5. Theoretical fit of the heating temperatures for zone $D\left(\approx 10 \mu \mathrm{m}\right.$ layer thickness, detached). $P=8 \mathrm{~W}, v_{L}=100 \mathrm{~Hz}$. Panel (a) presents the results for the $95^{\text {th }}$ laser pulse in the saturation regime for a mean temperature. Panel (b) presents the minimal and maximal heating temperatures for one hundred laser pulses on the whole temperature trace.

Table 1. The adjusted mean properties of the carbon layer deposited on the TEXTOR tile determined by active laser pyrometry. The accuracy of the results is mainly determined by the measurement accuracy of the layer thickness $d$ by optical microscopy.

\begin{tabular}{|c|c|c|c|}
\hline & Zone B & Zone C & $\begin{array}{c}\text { Zone D } \\
\text { (detached) }\end{array}$ \\
\hline$d[\mu \mathrm{m}]$ & $33 \pm 13$ & $4 \pm 2$ & 10 \\
\hline$\rho_{l}\left[\mathrm{~kg} / \mathrm{m}^{3}\right]$ & 650 & 1792 & 450 \\
\hline$C_{l}[\mathrm{~J} /(\mathrm{kg} \mathrm{K})]$ & 1500 & 1500 & 1500 \\
\hline$k_{l}[\mathrm{~W} /(\mathrm{m} \mathrm{K})]$ & 0.1 & 10 & 0.1 \\
\hline$\alpha_{l}\left[\mu \mathrm{m}^{-1}\right]$ & 1 & 2.13 & 1 \\
\hline$R_{l}$ & 0.7 & 0.23 & 0.9 \\
\hline$h_{\text {alp }}\left[\mathrm{kW} /\left(\mathrm{m}^{2} \cdot \mathrm{K}.\right)\right]$ & 3.925 & 14 & 0.325 \\
\hline$h_{p h}\left[\mathrm{~kW} /\left(\mathrm{m}^{2} \cdot \mathrm{K}.\right)\right]$ & $h \sim 10$ & $h \sim 10-100$ & $0.1<h<1.0$ \\
\hline
\end{tabular}

\subsection{Lock-in thermography}

Lock-in thermography was applied to determine thermal contact coefficient $\left(h_{p h}\right)$ by phase shifts measurements with different laser repetition rate frequencies. To determine the experimental phase shifts, the temperature evolution traces obtained by pyrometer measurements were used. Theoretical phase shifts were simulated with a new model for rapid calculations. Both the experimental and theoretical phase shifts versus laser repetition rate frequency for zones $B$, C, and D are presented in Fig. 6, Fig. 7, and Fig 8, respectively. The experimental results are given by points with errors bars. The theoretical phase shifts in these figures correspond to the Fourier component with $n=1$, that is, to the Fourier component on the laser repetition rate frequency $v_{L}$. The phase shift modelling was made with the adjusted layer properties obtained by laser active pyrometry (see Table 1), and layer/surface thermal contact coefficient $h_{p h}$ was an adjustable (variable) parameter. 


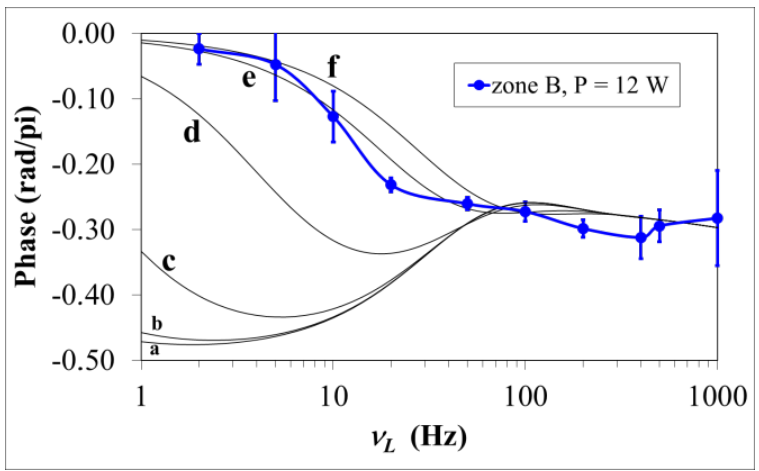

Fig. 6. Zone $B$. Simulated phase shifts (a-f) versus the repetition rate laser frequency $v_{L}$ for different $h\left(k W /\left(m^{2} . K\right)\right)$. For (a) $h \leq 0.001$, (b) $h=0.01$, (c) $h=0.1$, (d) $h=1$, (e) $h=10$, and (f) $h \geq 100$. The layer thickness in the simulations is $d=33 \mu \mathrm{m}$, and the laser beam radius $r_{0}=1000 \mu \mathrm{m} . P=12 \mathrm{~W}$.

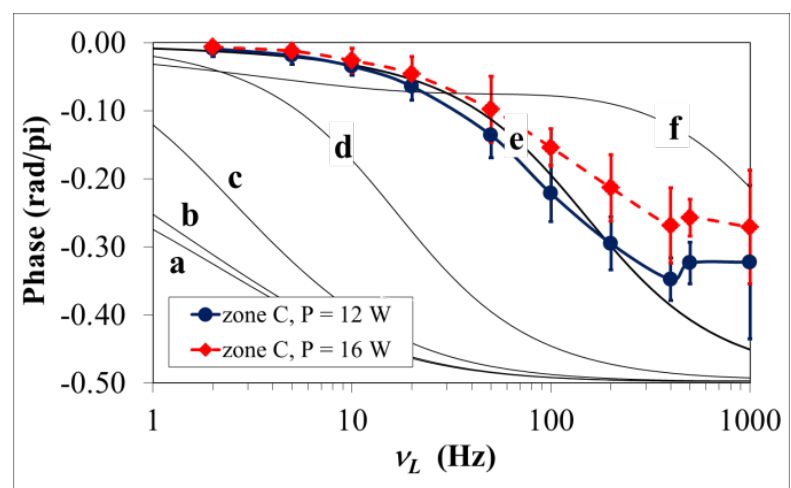

Fig. 7. Zone C. Simulated phase shifts (a-f) versus the repetition rate laser frequency $v_{L}$ for different $h\left(\mathrm{~kW} /\left(\mathrm{m}^{2} \cdot \mathrm{K}\right)\right)$. For (a) $h \leq 0.001$, (b) $h=0.01$, (c) $h=0.1$, (d) $h=1$, (e) $h=10$, and (f) $h \geq 100$. The layer thickness in the simulations is $d=4 \mu \mathrm{m}$, and the laser beam radius $r_{0}=1000 \mu \mathrm{m} . P=12 \mathrm{~W}$ and $16 \mathrm{~W}$.

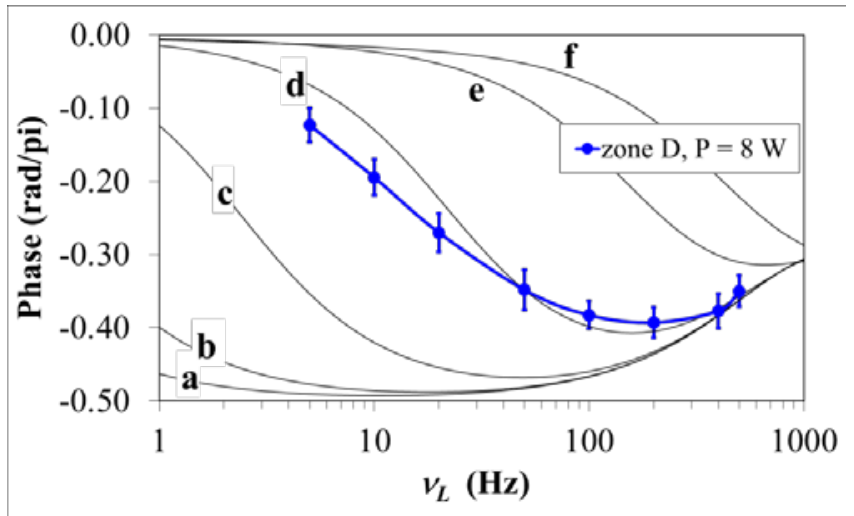

Fig. 8. Zone $D$. Simulated phase shifts (a-f) versus the repetition rate laser frequency $v_{L}$ for given $h\left(\mathrm{~kW} /\left(\mathrm{m}^{2} \cdot \mathrm{K}\right)\right)$. For (a) $h \leq 0.001$, (b) $h=0.01$, (c) $h=0.1$, (d) $h=1$, (e) $h=10$, and (f) $h \geq 100$. The layer thickness in the simulations is $d=10 \mu \mathrm{m}$, and the laser beam radius $r_{0}=1000 \mu \mathrm{m} . \quad P=8 \mathrm{~W}$.

From Figs. 6-8 for zones B, C, and D, the thermal contact coefficients are $h_{p h} \approx 10 \mathrm{~kW} /\left(\mathrm{m}^{2} \mathrm{~K}\right), h_{p h} \approx 10-100$ $\mathrm{kW} /\left(\mathrm{m}^{2} \cdot \mathrm{K}\right)$, and $h_{p h} \approx 1 \mathrm{~kW} /\left(\mathrm{m}^{2} \cdot \mathrm{K}\right)$, respectively (Table 1 , last line). From comparison of $h_{p h}$ with the thermal contact coefficients determined by active laser pyrometry $\left(h_{\text {alp }}\right)$, one may conclude that the results obtained by both methods are quite comparable. Though there was no gain in the measurements accuracy, lock-in measurements have demonstrated their advantage as being much more rapid. 
http://dx.doi.org/10.21611/qirt.2014.053

\section{Conclusions}

Active laser pyrometry and lock-in thermography were applied for in situ non-destructive characterization of micrometric layers on graphite substrates from European tokamaks [2-8]. The studies were aimed to obtain layer characterization with cross-checking the layer thermal contact coefficients determined by active laser pyrometry and lock-in thermography. The experimental installation comprised a Nd:YAG pulsed repetition rate lasers $(1 \mathrm{~Hz}-1 \mathrm{kHz}$ repetition rate frequency, homogeneous spot) and a home-made pyrometer system based on two pyrometers for the temperature consecutive measurements in 500 - $2600 \mathrm{~K}$ range. The study was aimed to cross-check the layer thermal contact coefficients obtained by active laser pyrometry and lock-in thermography.

Both for active laser pyrometry and lock-in thermography, the layer characterization was provided by the best fit of the experimental results and simulations. The experimental results obtained by active laser pyrometry were simulated by the developed 3D model for heating temperatures. The experimental phase shifts determined by lock-in thermography were simulated by the developed model for rapid phase shift calculations. The layer thermal contact coefficients determined by active laser pyrometry $\left(h_{a l p}\right)$ and those by lock-in thermography $\left(h_{p h}\right)$ were quite comparable. Though there was no gain in the measurements accuracy, lock-in measurements have proved their advantage as being much more rapid for determination of thermal contact coefficient for deposited layers of micrometric thickness.

To improve the lock-in thermography measurements of the phase shifts, the following considerations should be taken into account. Careful adjustment of the laser repetition rate is required. The laser pulse energy should not be too high to avoid any modifications in the layer properties resulting from the excessive heating of the surface layer. For the zones with a poor thermal contact, the rapid increase in the surface temperature may result in the layer surface modifications. In this case, it is not evident whether the measured phase shifts are attributed to different thermal contact quality or to the resulted layer modifications.

For surface properties characterization by lock-in thermography or active laser pyrometry, it may be advised to use a pyrometer of 330 - $1300 \mathrm{~K}$ temperature measurements range, which may provide measurements of a rather low temperature modulation of the surface under heating. At the low temperature modulation, the layer properties may be regarded as constant, and the phase shifts measurements are not sensitive to the layer properties dependence on a heating temperature. In addition, the undesirable surface oxidation may be avoided.

To decrease laser heating fluence, it may be advised to increase the laser spot diameter on the studied surface. For the temperature measurements in this case, the infrared camera rather than the pyrometer should be used. The infrared camera may offer the advantage of making the sample surface thermography and phase measurements simultaneously for each pixel of the camera.

Both the methods under discussion may be advised for applications in nuclear industry and art objects. For deposited layers on the surface, one may get information both on its mechanical properties (thickness, undersurface defects and cracks) and on thermo-physical ones (thermal conductivity and diffusivity, thermal resistance coefficient of the surface layer). However, up to now, the potentialities of the methods are restricted to micrometric layers of thickness exceeding roughly $1 \mu \mathrm{m}$. Applicability of the methods for characterization of very thin $(<1 \mu \mathrm{m})$ layers requires additional studies.

\section{REFERENCES}

[1] Brygo F., Semerok A., Oltra R., Weulersse J.-M. and Fomichev S.V., "Laser heating and ablation at high repetition rate in thermal confinement regime," Appl. Surf. Sci., vol. 252, pp. 8314-8318, 2006.

[2] Semerok A., Fomichev S.V., Weulersse J.-M., Brygo F., Thro P.-Y. and Grisolia C., "Heating and ablation of tokamak graphite by pulsed nanosecond Nd-YAG lasers," J. Appl. Phys., vol. 101, 084916, 2007.

[3] Grisolia C., Semerok A., Weulersse J.-M., Le Guern F., Fomichev S., Brygo F., Fichet P., Thro P.-Y., Coad P., Bekris N., Stamp M., Rosanvallon S., and Piazza G., "In-situ tokamak laser applications for detritiation and codeposited layers studies," J. Nucl. Mater., vol. 363-365, pp.1138-1147, 2007.

[4] Courtois X., Sortais C., Melyukov D., Gardarein J.-L., Semerok A. and Grisolia C., "Development of laser lock-in thermography for plasma facing component surface characterisation," Fusion Eng. Des., vol. 86, pp. $1714-$ 1718, 2011.

[5] Semerok A., Jaubert F., Fomichev S.V., Thro P.-Y., Courtois X. and Grisolia C., "Laser lock-in thermography for thermal contact characterisation of surface layer," Nucl. Instrum. and Meth. A, vol. 693, pp. 98-103, 2012.

[6] Semerok A., Fomichev S.V., Brygo F., Thro P.-Y. and Grisolia C., "Pulsed repetition rate nanosecond laser heating and ablation of the tokamak graphite tile deposited layers," J. Nucl. Mater., vol. 420, pp. 198-206, 2012.

[7] Semerok A., Jaubert F., Fomichev S.V., Thro P.-Y. and Grisolia C., "Tokamak active laser pyrometry for tungsten deposited layer characterization," Fusion Eng. Des., vol. 87, pp. 267-274, 2012.

[8] Semerok A., Fomichev S.V., Jaubert F., Grisolia C., "Active laser pyrometry and lock-in thermography for characterisation of deposited layer on TEXTOR graphite tile", Nucl. Instrum. and Meth. A, vol. 738, pp. 25-33, 2014.

[9] Carslaw H.S. and Jaeger J.C., Conduction of Heat in Solids, 2nd ed., Oxford University Press, Oxford, 1959.

[10] Landau L.D., Lifshitz E.M. and Pitaevskii L.P., Electrodynamics of Continuous Media, 2nd edition, Oxford, Butterworth-Heinemann, 1995. 